\title{
Effects of Different Methionine Levels in Low Protein Diets on Production Performance, Reproductive System, Metabolism, and Gut Microbiota in Laying Hens
}

\author{
Miaolin $\mathrm{Ma}^{\dagger}$, Shunju Geng ${ }^{\dagger}$, Meiling Liu, Lihong Zhao, Jianyun Zhang, Shimeng Huang ${ }^{*}$ \\ and Qiugang $\mathrm{Ma}^{*}$ \\ State Key Laboratory of Animal Nutrition, College of Animal Science and Technology, China Agricultural University, Beijing, \\ China
}

OPEN ACCESS

Edited by:

Tingtao Chen,

Nanchang University, China

Reviewed by:

Chunqi Gao,

South China Agricultural

University, China

Huan $L i$

Lanzhou University, China Xinyang Dong,

Zhejiang University, China

*Correspondence:

Qiugang Ma

maqiugang@cau.edu.cn

Shimeng Huang

shimengh@hotmail.com

†These authors have contributed equally to this work

Specialty section

This article was submitted to Nutrition and Microbes,

a section of the journal

Frontiers in Nutrition

Received: 11 July 2021 Accepted: 06 September 2021 Published: 06 October 2021

Citation:

Ma M, Geng S, Liu M, Zhao L, Zhang J, Huang S and Ma Q (2021) Effects of Different Methionine Levels in Low Protein Diets on Production Performance, Reproductive System, Metabolism, and Gut Microbiota in Laying Hens. Front. Nutr. 8:739676. doi: 10.3389/fnut.2021.739676
This study investigated the effects of different levels of methionine (Met) in a low protein diet on the production performance, reproductive system, metabolism, and gut microbial composition of laying hens to reveal the underlying molecular mechanism of Met in a low protein diet on the host metabolism and gut microbial composition and function of hens. A total of 360 healthy 38-week-old Peking Pink laying hens with similar body conditions and egg production (EP) were randomly divided into four groups with nine replicates per treatment and 10 hens per replicate. The hens in each treatment group were fed low protein diets containing different levels of Met $(0.25,0.31,0.38$, and $0.47 \%$, respectively) for 12 weeks. Feed and water were provided ad libitum throughout the trial period. The results showed that, compared with the $0.25 \%$ Met group, the final body weight (FBW), average daily gain (ADG), EP, egg weight (EW), and average daily feed intake (ADFI) in the other groups were significantly increased and feed egg ratio (FER) was decreased. Meanwhile, the EW and yield of abdominal fat (AFY) in the $0.47 \%$ Met group were higher than those in other groups. The triglyceride (TG), estradiol (E2), total protein (TP), albumin (ALB), and immunoglobulin A (IgA) in the 0.38 and $0.47 \%$ Met groups were higher than those in other groups. In addition, $16 \mathrm{~S}$ rRNA gene sequencing revealed that there was no difference in the Sobs index, ACE index, and Shannon index among all groups. However, it is worth noting that feeding low protein diets with Met changed the gut microbial composition (e.g., the supplementation of Met increased the level of Lactobacillus and decreased the proportion of Faecalibacterium). Also, our results showed that the changes in gut microbial composition induced by the diets with different levels of Met were closely related to the changes of key parameters: ADFI, EW, FBW, TG, EM, EP, ADG, FER, and uric acid (UA). Our results highlight the role of adding an appropriate amount of Met to the low protein diet in laying hens, which could improve the gut microbial composition, production performance, reproductive system, and nutrient metabolism of laying hens. In conclusion, this study suggested that when the Met level was $0.38 \%$, the production performance of the laying hens was pretty good.

Keywords: laying hens, methionine deficiency, low protein diets, gut microbiota, production performance, nutrient metabolism 


\section{INTRODUCTION}

The poultry industry shows a trend of large-scale, intelligent, and standardized development, and the demand for protein feed resources continues to increase $(1,2)$. However, there are problems, such as limited protein resources, low nutrient utilization rates, and environmental pollution, caused by fecal nitrogen emission, thereby making the research on the low protein diet is becoming more and more important. In order to ensure the performance of animals, a large amount of protein is added to the diet of laying hens. However, excessive protein in the diet, which will increase the content of nitrogen and phosphorus in feces and urine, is often not put to good use $(3,4)$. Excessive ammonia nitrogen emission in feces will not only cause environmental pollution, but also harm the health of laying hens (5-8). Some studies have shown that reducing protein levels in the diet can effectively reduce nitrogen emissions from the feces and urine of livestock and poultry (9-11).

That refers to the low protein diet that containing less crude protein than the nutrient requirements of poultry (NRC) recommends for feeding in the diet. Following the ideal amino acid pattern is the key to feed formulation design. In essence, the need for protein in the diet is the need for amino acids, which are essential nutrients for animal growth and development that are involved in important biochemical reactions in animals $(12,13)$. There have also been studies on livestock and poultry that have shown that adding certain synthetic amino acids to a low protein diet can improve protein digestibility, effectively reduce nitrogen emissions $(11,14)$, and save feed costs $(15,16)$. Many studies have shown that adding a certain amount of glycine, valine, isoleucine, and methionine (Met) to a low protein diet has no negative effect on livestock and poultry and can even improve their performance in some way (17-19). In addition, it can also improve the structure and composition of the gut microbiota of livestock and poultry, increase the abundance of beneficial microbes such as Bifidobacterium and Lactobacillus, maintain the integrity of the intestinal epithelial barrier function, improve the normal functions of the intestinal epithelium, and enhance intestinal mucosal immunity in animals (20-23).

In order to avoid the negative effects of a low protein diet on livestock and poultry, we can consider adding essential amino acids to their diet to reduce harmful effects (24). Methionine, as the main limiting amino acid in the corn-soybean meal diet of laying hens, not only participates in protein synthesis, but also plays an irreplaceable role in some molecular precursors and intermediates to control oxidative stress in the body and affect cell metabolism and function (25). It was also found that dietary Met can enhance the immune response levels of poultry $(26,27)$. Furthermore, Met can be added into the low protein diet of broilers to meet the requirements of total sulfur-containing amino acids, but it should be added appropriately, otherwise, it

Abbreviations: ADG, average daily gain; ADFI, average daily feed intake; AFY, yield of abdominal fat; ALB, albumin; BW, body weight; EP, egg production; EW, egg weight; EM, average daily egg mass; FBW, final body weight; FER, feed egg ratio; GSH, glutathione; IBW, initial body weight; IgM, immune globulin M; Met, methionine; SOD, superoxide dismutase; TG, triglyceride; TP, total protein; UA, uric acid. will affect the growth performance of poultry (28). Zhang found that supplemented Met in the diet could change the intestinal tissue morphology and increase the body weight of Peking ducks (29). In addition, Met can provide methyl $(30,31)$, and use it for the methylation of toxic substances to detoxify. It can also combine with mycotoxin to weaken its toxicity. But there is still a lack of research on the effect of Met supplementation in low protein diets on laying hens, especially on the effect of gut microbiota.

Due to the current situation, low protein diets will be a trend in the future. However, the application of a low crude protein diet in laying hens is relatively rare at present, and the study on its functional effect on laying hens can better prepare balanced low protein diets, which is conducive to exerting the genetic potential of laying hens. To develop a practical understanding of Met use in low crude protein diets, a study was conducted to evaluate production performance, egg quality, reproductive system, host metabolism, and the gut microbial composition responses to different levels of Met in laying hens fed low protein diets.

\section{MATERIALS AND METHODS}

\section{Animal Experimental Ethics}

All experiments were approved by the China Agricultural University Animal Care and Use Committee (AW32301202-2-1, Beijing, China).

\section{Animals and Experimental Design}

A total of 360 commercial hens of the Peking Pink strain (Yukou Poultry Co., Ltd., Beijing, China) at the age of 38 weeks with similar egg production and healthy bodies were randomly divided into four treatment groups with nine replicates per group and 10 birds per replicate. The hens were placed in five cages (two birds in each cage) and each cage ( $\mathrm{H} 45 \mathrm{~cm} \times \mathrm{W} 45 \mathrm{~cm} \times$ $\mathrm{D} 45 \mathrm{~cm}$ ) was equipped with a nipple drinker and an exterior feed through to ensure feed and water were provided ad libitum during the entire experimental period. At the same time, to ensure that the chicken coop is closed and ventilated, the average relative humidity was routinely maintained at $\sim 55 \%$, and it was ensured that the hens get $16 \mathrm{~h}$ of light every day. In order to meet the nutritional requirements of the laying hens (NYT332004), a basal corn-soybean meal diet was formulated. A preexperiment was conducted for one week before the start of the formal experiment to ensure that the animals were acclimated to the new experimental environment and diet, meanwhile, it could empty the original intestinal contents, and estimate the approximate feed intake of the experimental animals. The four experimental groups were fed with low protein diets containing $0.25,0.31,0.38$, and $0.47 \%$ Met $(0.25 \%$ Met group, $0.31 \%$ Met group, $0.38 \%$ Met group, and $0.47 \%$ Met group), respectively. The ingredients and nutrient composition of the diets are shown in Supplementary Table 1.

\section{Laying Performance and Egg Quality}

The difference between the full bucket weights and the remaining feed was calculated as the weekly feed intake, and the body weights of the laying hens were recorded every week at the 
same time to calculate the average daily feed intake (ADFI) and feed egg ratio (FER). The number of eggs and egg weight were accurately recorded every day to calculate the average daily egg mass (EM), egg weight (EW), and egg production (EP) rate. The hens were weighed in replicates at the beginning and end of the experiment to calculate average daily gain (ADG). After the beginning of the experiment, 30 eggs were randomly selected from each treatment group every 4 weeks to measure egg quality parameters as shown in Table 2.

The eggshell strength was measured by the egg force reader (ESTG-01ĄCOrka Teachnology Ltd); The eggshell thickness was measured using the eggshell thickness tester (ESTG-01, Orka Teachnology Ltd); Haugh unit, yolks color, and egg weight were measured by multifunctional egg quality tester (EA-01, Orka Technology Ltd). The eggshell color was measured by QCR color reflectometer (QCR SPA, TSS England). Weigh the eggshell, then separate the yolk with a separator, and then weigh the ratio of yolk and the ratio of albumen.

\section{Blood Sampling and Biochemical Analysis}

At the end of the experiment, blood samples were collected from the wing veins of the laying hens on the same day of sampling and centrifuged at 3,000 rpm for $15 \mathrm{~min}$ at room temperature to separate the serum. After that, the serum samples were collected by a pipette into $1.5-\mathrm{ml}$ tubes and stored at $-20^{\circ} \mathrm{C}$. Triglyceride (TG), uric acid (UA), urea, total protein (TP), albumin (ALB), and immunoglobulin $\mathrm{M}(\operatorname{IgM})$ in serum were determined using an automatic biochemical analyzer (7600, Hitachi, Japan) according to the manufacturer's instructions. Superoxide dismutase (SOD) and glutathione (GSH) in the serum were determined using a commercial kit (Nanjing Jiancheng Bioengineering Institute, Nanjing, China) according to the kit instructions.

\section{Collecting Samples}

Seventy hens were euthanized and weighed by a sodium pentobarbital injection $(0.4 \mathrm{ml} / \mathrm{kg} \cdot \mathrm{BW}$; Sile Biological Technology Co., Ltd., Guangzhou, China). Abdominal adipose tissue was weighed to calculate the yield of abdominal fat (AFY). The liver, kidneys, fallopian tubes, and ovaries were removed, weighed, and the number of follicles was recorded to calculate the liver index, fallopian tube index, and ovary index.

\section{DNA Extraction, Amplification, and Sequencing}

The cecal contents of laying hens were collected, immediately frozen in liquid nitrogen, and stored at $-80^{\circ} \mathrm{C}$. Cecal microbial DNA was isolated with an Omega Bio-tek stool DNA kit (Omega, Norcross, GA, USA) and quantified by a NanoDrop 2000 spectrophotometer (Thermo Scientific, Waltham, MA, USA). Then, the V3-V4 region of the $16 \mathrm{~S}$ rRNA gene was amplified with $338 \mathrm{~F}$ and 806R primers with the sequence of $5^{\prime}$-ACTCCTACGGGAGCAGCA-3' and $5^{\prime}$ GGACTACHVGGGTWTCTAAT-3'. Afterward, DNA samples were quantified, followed by the amplification of V3V4 hypervariable region of the $16 \mathrm{~S}$ rDNA. Final amplicon pool was evaluated by the AxyPrep DNA gel extraction kit (Axygen
Biosciences, Union City, CA, USA). Paired-end reads were generated with an Illumina MiSeq PE250 (Shanghai MajorBio Biopharma Technology Co., Ltd., Shanghai, China), and the reads were filtered out with default parameters.

\section{Statistical Analysis}

All results were subjected to a one-way ANOVA procedure and differences were examined using Duncan's multiple range test to evaluate the differences within treatments using SPSS version 18.0 (SPSS Institute Inc., Chicago, USA). The trends of the linear and quadratic analyses were conducted using SAS software version 8.0 (version 9.2, SAS Institute Inc., Cary, NC, USA). Differences were considered significant at $p<0.05$. Data were expressed as the mean $\pm \mathrm{SE}$.

The raw paired-end reads were assembled into longer sequences, and quality was filtered by PANDAseq (version 2.9) to remove the low-quality reads. The high-quality sequences were clustered into operational taxonomic units (OTUs) with a 97\% similarity using UPARSE (version 7.0) in QIIME (version 1.17) $(32,33)$, and the chimeric sequences were removed using UCHIME (34). Taxonomy was assigned to OTUs using the RDP classifier. The subsequent clean reads were clustered as OTUs using UPARSE (version 7.0) and annotated with the SILVA 16S rRNA gene database using the MOTHUR program (version v.1.30.1) (35). Alpha-diversity (the Chao index, Ace index, and Sob index) was calculated based on the profiles of OTU by the MOTHUR program (36). Bar plots and heat maps were generated with the "vegan" package in $\mathrm{R}$ (version 3.3.1). A principal coordinate analysis (PCoA) was performed based on the Bray-Curtis distance using QIIME (version 1.17). An analysis of similarities (ANOSIM) was performed to compare the similarity of bacterial communities among groups using the "vegan" package of $\mathrm{R}$ (version 3.3.1). A linear discriminant analysis (LDA) effect size (LEfSe) was performed to identify the bacterial taxa that are differentially enriched in different bacterial communities. In a redundancy analysis (RDA), the variance between the samples (genus-level relative bacterial abundance) is explained by the phenotype of laying hens, which were fitted to corresponding matrices in the resulting illustration (37-39). Phylogenetic Investigation of Communities by Reconstruction of Unobserved States (PICRUSt) was also used to obtain a deeper insight into different pathways based on the Kyoto Encyclopedia of Genes and Genomes (KEGG) orthology between the four groups (40). Finally, the correlations between key parameters and bacterial communities were assessed by Spearman's correlation analysis using the "pheatmap" package in R (version 3.3.1). Data were expressed as mean values.

\section{RESULTS}

\section{Effects of Different Levels of Met Supplementation on the Laying Performance of Laying Hens With Low Protein Diets}

During the experiment period (Table 1), dietary Met levels had significant positive effects on the performance of laying hens. 
TABLE 1 | Effects of different dietary Met supplementation in low protein diets on the growth and laying performance of laying hens that are 38-50 weeks.

\begin{tabular}{|c|c|c|c|c|c|c|c|c|}
\hline Indexes & $\begin{array}{c}0.25 \% \\
\text { Met group }\end{array}$ & $\begin{array}{c}0.31 \% \\
\text { Met group }\end{array}$ & $\begin{array}{c}0.38 \% \\
\text { Met group }\end{array}$ & $\begin{array}{c}0.47 \% \\
\text { Met group }\end{array}$ & $\mathrm{SE}^{\mathrm{d}}$ & $P$-value & Linear $P$-value & Quadratic $P$-value \\
\hline IBW (g) & $1,507.3$ & $1,507.6$ & $1,502.9$ & 1,512 & 15.77 & 0.982 & 0.896 & 0.78 \\
\hline FBW (g) & $1,509.9^{b}$ & $1,647.2^{\mathrm{a}}$ & $1,629.2^{a}$ & $1,652.9^{a}$ & 17.94 & $<0.001$ & $<0.001$ & 0.003 \\
\hline$A D G(g / d)$ & $0.03^{b}$ & $1.70^{\mathrm{a}}$ & $1.54^{\mathrm{a}}$ & $1.72^{\mathrm{a}}$ & 0.19 & $<0.001$ & $<0.001$ & $<0.001$ \\
\hline EP (\%) & $68.1^{\mathrm{b}}$ & $83.9^{\mathrm{a}}$ & $87.4^{a}$ & $86.9^{\mathrm{a}}$ & 1.47 & $<0.001$ & $<0.001$ & $<0.001$ \\
\hline EW (g) & $56.4^{c}$ & $60.6^{a, b}$ & $60.1^{\mathrm{b}}$ & $61.7^{a}$ & 0.39 & $<0.001$ & $<0.001$ & 0.002 \\
\hline EM $(g / d)$ & $38.4^{b}$ & $50.8^{a}$ & $52.5^{\mathrm{a}}$ & $53.5^{a}$ & 0.87 & $<0.001$ & $<0.001$ & $<0.001$ \\
\hline ADFI $(g / d)$ & $97.4^{\mathrm{b}}$ & $110.2^{a}$ & $109.6^{a}$ & $110.7^{a}$ & 1.3 & $<0.001$ & $<0.001$ & $<0.001$ \\
\hline FER & $2.54^{\mathrm{a}}$ & $2.17^{b}$ & $2.09^{b}$ & $2.07^{b}$ & 0.03 & $<0.001$ & $<0.001$ & $<0.001$ \\
\hline AFY (\%) & $1.32^{b}$ & $2.52^{a, b}$ & $3.29^{a}$ & $3.08^{a}$ & 0.36 & 0.002 & 0.001 & 0.055 \\
\hline Liver index (\%) & $2.64^{a}$ & $2.35^{a, b}$ & $2.23^{b}$ & $2.29^{a, b}$ & 0.1 & 0.037 & 0.014 & 0.104 \\
\hline
\end{tabular}

a,b,c Means within a column with no common superscripts differ $(p<0.05)$

${ }^{d}$ Pooled SEM.

TABLE 2 | The effects of different dietary met supplementation in low protein diets on the egg quality of 42-, 46-, and 50-week-old laying hens.

\begin{tabular}{|c|c|c|c|c|c|c|c|c|c|}
\hline \multirow[t]{2}{*}{ Indexes } & \multirow[t]{2}{*}{ Age } & \multirow{2}{*}{$\begin{array}{c}0.25 \% \\
\text { Met group }\end{array}$} & \multirow{2}{*}{$\begin{array}{c}0.31 \% \\
\text { Met group }\end{array}$} & \multirow{2}{*}{$\begin{array}{c}0.38 \% \\
\text { Met group }\end{array}$} & \multirow{2}{*}{$\begin{array}{c}0.47 \% \\
\text { Met group }\end{array}$} & \multirow[t]{2}{*}{$\mathbf{S E}^{\mathrm{C}}$} & \multicolumn{3}{|c|}{$P$-value } \\
\hline & & & & & & & Total & Linear & Quadratic \\
\hline \multirow[t]{3}{*}{ Egg weight (g) } & 42 & $55.90^{b}$ & $59.94^{a}$ & $60.24^{a}$ & $61.90^{a}$ & 0.73 & $<0.001$ & $<0.001$ & 0.102 \\
\hline & 46 & $58.47^{b}$ & $61.67^{a}$ & $61.20^{a, b}$ & $61.70^{a}$ & 0.84 & 0.019 & 0.014 & 0.107 \\
\hline & 50 & $56.48^{b}$ & $60.23^{a}$ & $60.60^{a}$ & $61.61^{a}$ & 0.76 & $<0.001$ & $<0.001$ & 0.073 \\
\hline \multirow[t]{3}{*}{ Shell (\%) } & 42 & 10.94 & 10.81 & 10.8 & 10.63 & 0.15 & 0.523 & 0.154 & 0.922 \\
\hline & 46 & $10.95^{a}$ & $10.10^{b}$ & $10.93^{a, b}$ & $10.70^{a, b}$ & 0.23 & 0.033 & 0.930 & 0.177 \\
\hline & 50 & 10.8 & 10.8 & 10.72 & 10.37 & 0.19 & 0.345 & 0.118 & 0.366 \\
\hline \multirow[t]{3}{*}{ Albumen (\%) } & 42 & $62.36^{b}$ & $63.68^{a}$ & $63.53^{a, b}$ & $63.36^{a, b}$ & 0.32 & 0.025 & 0.056 & 0.026 \\
\hline & 46 & 63.56 & 65.55 & 63.17 & 62.83 & 1.06 & 0.270 & 0.340 & 0.280 \\
\hline & 50 & $61.37^{b}$ & $62.95^{a, b}$ & $62.32^{a, b}$ & $63.62^{a}$ & 0.54 & 0.026 & 0.012 & 0.79 \\
\hline \multirow[t]{3}{*}{ Yolk (\%) } & 42 & $26.69^{a}$ & $25.50^{b}$ & $25.68^{a, b}$ & $26.01^{a, b}$ & 0.28 & 0.018 & 0.138 & 0.008 \\
\hline & 46 & 25.53 & 24.20 & 25.82 & 26.47 & 1 & 0.417 & 0.315 & 0.323 \\
\hline & 50 & 27.82 & 26.27 & 26.96 & 26.01 & 0.52 & 0.071 & 0.045 & 0.560 \\
\hline \multirow[t]{3}{*}{ Shell color } & 42 & 0.58 & 0.57 & 0.56 & 0.55 & 0.01 & 0.337 & 0.070 & 0.913 \\
\hline & 46 & 55.89 & 56.53 & 54.82 & 56.98 & 0.95 & 0.417 & 0.711 & 0.424 \\
\hline & 50 & 0.46 & 0.46 & 0.46 & 0.44 & 0.01 & 0.101 & 0.135 & 0.045 \\
\hline \multirow[t]{3}{*}{ Shell thickness (mm) } & 42 & 0.58 & 0.57 & 0.56 & 0.55 & 0.01 & 0.337 & 0.070 & 0.913 \\
\hline & 46 & $0.46^{b}$ & $0.46^{b}$ & $0.48^{a}$ & $0.46^{b}$ & 0.01 & 0.001 & 0.189 & 0.229 \\
\hline & 50 & 0.46 & 0.46 & 0.46 & 0.44 & 0.01 & 0.101 & 0.135 & 0.045 \\
\hline \multirow[t]{3}{*}{ Shell strength (N) } & 42 & 41.02 & 42.72 & 40.49 & 39.75 & 1.27 & 0.384 & 0.286 & 0.332 \\
\hline & 46 & 40.98 & 40.9 & 41.25 & 40.61 & 1.32 & 0.989 & 0.896 & 0.835 \\
\hline & 50 & 38.25 & 40.5 & 38.13 & 36.57 & 1.48 & 0.326 & 0.267 & 0.204 \\
\hline \multirow[t]{3}{*}{ Haugh unit } & 42 & 84.49 & 83.23 & 82.96 & 83.47 & 1.03 & 0.740 & 0.471 & 0.388 \\
\hline & 46 & 85.85 & 84.00 & 80.41 & 83.38 & 1.80 & 0.216 & 0.168 & 0.182 \\
\hline & 50 & 82.23 & 85.58 & 81.61 & 82.53 & 1.89 & 0.466 & 0.717 & 0.523 \\
\hline \multirow[t]{3}{*}{ Yolk color } & 42 & 4.86 & 4.77 & 4.83 & 4.60 & 0.10 & 0.240 & 0.100 & 0.500 \\
\hline & 46 & 4.78 & 4.71 & 4.80 & 4.83 & 0.11 & 0.897 & 0.630 & 0.680 \\
\hline & 50 & $4.73^{\mathrm{a}}$ & $4.27^{b}$ & $4.43^{a, b}$ & $4.70^{a, b}$ & 0.13 & 0.029 & 0.906 & 0.004 \\
\hline
\end{tabular}

a,b Means within a column with no common superscripts differ $(p<0.05)$.

${ }^{c}$ Pooled SEM.

There were no significant differences in FBW, ADG, EP, EW, but they were significantly higher than those in the $0.25 \%$ Met $\mathrm{EM}$, and ADFI between the $0.31,0.38$, and $0.47 \%$ Met groups, group $(p<0.001)$. On the contrary, the FER in the $0.25 \%$ Met 
TABLE 3 | The effects of different dietary Met supplementation in low protein diets on the serum parameters of laying hens that are 38-50 weeks.

\begin{tabular}{|c|c|c|c|c|c|c|c|c|}
\hline Indexes & $\begin{array}{c}0.25 \% \\
\text { Met group }\end{array}$ & $\begin{array}{c}0.31 \% \\
\text { Met group }\end{array}$ & $\begin{array}{c}0.38 \% \\
\text { Met group }\end{array}$ & $\begin{array}{c}0.47 \% \\
\text { Met group }\end{array}$ & $\mathrm{SE}^{\mathrm{C}}$ & $P$-value & $\begin{array}{l}\text { Linear } \\
P \text {-value }\end{array}$ & $\begin{array}{c}\text { Quadratic } \\
P \text {-value }\end{array}$ \\
\hline TG (mmol/L) & $7.64^{b}$ & $8.51^{b}$ & $10.56^{a}$ & $11.54^{a}$ & 0.39 & $<0.001$ & $<0.001$ & 0.89 \\
\hline UA ( $\mu \mathrm{mol} / \mathrm{L})$ & $82.33^{a}$ & $65.00^{b}$ & $67.22^{a, b}$ & $64.56^{b}$ & 4.48 & 0.03 & 0.02 & 0.11 \\
\hline Urea (mmol/L) & 0.31 & 0.32 & 0.33 & 0.37 & 0.02 & 0.31 & 0.09 & 0.45 \\
\hline TP (g/L) & 37.47 & 35.23 & 39.52 & 39.28 & 1.6 & 0.22 & 0.18 & 0.54 \\
\hline ALB (g/L) & $12.81^{a, b}$ & $11.41^{b}$ & $13.62^{a}$ & $13.00^{a, b}$ & 0.42 & 0.006 & 0.15 & 0.36 \\
\hline $\mathrm{P}(\mathrm{ng} / \mathrm{mL})$ & 0.84 & 0.84 & 1.05 & 1.13 & 0.19 & 0.61 & 0.21 & 0.85 \\
\hline E3 (pg/mL) & $101.03^{a, b}$ & $83.91^{b}$ & $165.41^{a}$ & $172.78^{a}$ & 18.74 & 0.003 & 0.001 & 0.52 \\
\hline $\lg G(g / L)$ & 5.98 & 6.19 & 6.08 & 6.53 & 0.27 & 0.52 & 0.21 & 0.67 \\
\hline $\lg A(g / L)$ & $1.06^{a, b}$ & $0.99^{b}$ & $1.04^{a, b}$ & $1.12^{\mathrm{a}}$ & 0.03 & 0.04 & 0.09 & 0.02 \\
\hline $\operatorname{lgM}(\mathrm{g} / \mathrm{L})$ & 0.8 & 0.83 & 0.79 & 0.89 & 0.03 & 0.15 & 0.11 & 0.32 \\
\hline
\end{tabular}

${ }^{a, b}$ Means within a column with no common superscripts differ $(p<0.05)$.

${ }^{c}$ Pooled SEM.

group was significantly higher than the other three groups ( $p$ $<0.001$ ), while there was no significant difference among the other three groups. The AFY in the 0.47 and $0.38 \%$ Met groups was significantly higher than that in the $0.25 \%$ Met group ( $p$ $<0.01$ ). The EW in the $0.38 \%$ Met group was significantly lower than that in the $0.47 \%$ Met group $(p<0.001)$, the liver index was lower than that in the $0.25 \%$ Met group $(p<$ 0.05 ), and IBW was not significantly affected by different dietary Met levels (Table 1).

When the diet was supplemented with $0.38 \%$ Met, the maximum EP $(88.83 \%)$ was obtained. When the Met level was increased to $0.41 \%$, the maximum EM $(54.32 \mathrm{~g} / \mathrm{d})$ and minimum FER (2.04) were obtained, and the Met intake was $0.45,0.46$, and $0.46 \mathrm{~g} / \mathrm{d}$, respectively (Supplementary Table 2). When the dietary sulfur amino acid content was $0.62 \%$, the maximum EP (88.28\%) was obtained. When the diet contained $0.63 \%$ sulfur amino acids, the maximum EM (53.98 g/d) and the minimum FER (2.05) were obtained. At this time, the intake of sulfur amino acids was $0.69,0.7$, and $0.7 \mathrm{~g} / \mathrm{day}$, respectively (Supplementary Table 2).

\section{Effects of Different Levels of Met Supplementation on the Egg Quality of Laying Hens With Low Protein Diets}

As shown in Table 2, the EW in the $0.25 \%$ Met group at 42 and 50 weeks of age was significantly lower than that of the other three groups $(p<0.001)$. The EW at 46 weeks of age in the $0.25 \%$ Met group was lower than that in the 0.31 and $0.47 \%$ Met groups $(p<0.05)$. The eggshell proportion at 46 weeks of age in the $0.25 \%$ Met group was higher than that in the $0.31 \%$ Met group $(p<0.05)$. The albumen ratio at 42 weeks of age in the $0.25 \%$ Met group was lower than that in the $0.31 \%$ Met group $(p<0.05)$, while the albumen ratio at 50 weeks of age was lower than that in the $0.47 \%$ Met group $(p<0.05)$. On the contrary, the yolk ratio at 42 weeks of age in the $0.25 \%$ Met group was higher than that in the $0.31 \%$ Met group $(p<0.05)$. The eggshell thickness in the $0.38 \%$ Met group at 46 weeks of age was significantly higher than that in the other three groups $(p<0.01)$, and there was no significant difference among the other three groups. The yolk color value in the $0.31 \%$ Met group at 50 weeks of age was the lowest and lower than that of the $0.25 \%$ Met group $(p<0.05)$. However, other indicators such as eggshell color, eggshell strength, and Haugh unit were not significantly affected by the different levels of dietary Met (Table 2).

\section{Effects of Different Levels of Met Supplementation on the Host Metabolism of Laying Hens With Low Protein Diets}

The different levels of dietary Met had significant effects on a number of biochemical indices (e.g., TG, ALB, UA, E2, and IgA) in the serum of laying hens. In particular, the TG and E2 in the $0.47 \%$ Met group were significantly higher than those in the $0.31 \%$ Met group $(p<0.01)$. Furthermore, IgA was higher than that in the $0.31 \%$ Met group $(p<0.05)$, while the TG, ALB, and E2 in the $0.38 \%$ Met group were significantly higher than those in the $0.31 \%$ Met group $(p<0.01)$. The TG in the $0.25 \%$ Met group was significantly lower than that in the 0.38 and $0.47 \%$ Met groups $(p<0.001)$, and UA was higher than that in the 0.31 and $0.47 \%$ Met groups $(p<$ 0.05). However, other biochemical indices were not significantly affected by different dietary Met levels (e.g., UREA, TP, P, IgG, and IgM) (Table 3).

\section{Effects of Different Levels of Met Supplementation on the Reproductive System of Laying Hens With Low Protein Diets}

As shown in Table 4, the different levels of dietary Met have extremely significant effects on tubal weight. The tubal weight of laying hens in the $0.47 \%$ Met group was significantly higher than that in the $0.25 \%$ Met group $(p<0.01)$ but it had no significant effects on oviduct length, ovary weight, and ovary weight after the removal of dominant follicles, etc., (Table 4). 
TABLE 4 | The effects of different dietary Met supplementation in low protein diets on the reproductive system of laying hens that are $38-50$ weeks.

\begin{tabular}{|c|c|c|c|c|c|c|c|c|}
\hline Indexes & $\begin{array}{c}0.25 \% \\
\text { Met group }\end{array}$ & $\begin{array}{c}0.31 \% \\
\text { Met group }\end{array}$ & $\begin{array}{c}0.38 \% \\
\text { Met group }\end{array}$ & $\begin{array}{c}0.47 \% \\
\text { Met group }\end{array}$ & $\mathrm{SE}^{\mathrm{c}}$ & $P$-value & $\begin{array}{c}\text { Linear } \\
P \text {-value }\end{array}$ & $\begin{array}{c}\text { Quadratic } \\
P \text {-value }\end{array}$ \\
\hline Oviduct weight (g) & $43.8^{b}$ & $49.7^{a, b}$ & $50.7^{a, b}$ & $55.3^{a}$ & 2.19 & 0.009 & 0.001 & 0.774 \\
\hline Oviduct length (cm) & 67.9 & 73.9 & 71 & 71.6 & 2.4 & 0.383 & 0.444 & 0.278 \\
\hline Ovary weight (g) & 33.1 & 34.3 & 35.6 & 42.3 & 2.5 & 0.061 & 0.014 & 0.28 \\
\hline Ovarian weight except the dominant follicles (g) & 5.3 & 5.7 & 5.6 & 5.3 & 0.6 & 0.937 & 0.976 & 0.535 \\
\hline The number of rhubarb follicles & 3.8 & 3.2 & 3.3 & 3.9 & 0.2 & 0.072 & 0.63 & 0.01 \\
\hline The number of small yellow follicles & 1 & 1.2 & 1.2 & 1 & 0.1 & 0.227 & 1 & 0.04 \\
\hline The number of white follicles & 0.9 & 0.9 & 0.9 & 1.4 & 0.29 & 0.455 & 0.214 & 0.352 \\
\hline
\end{tabular}

${ }^{a, b}$ Means within a column with no common superscripts differ $(p<0.05)$.

${ }^{c}$ Pooled SEM.
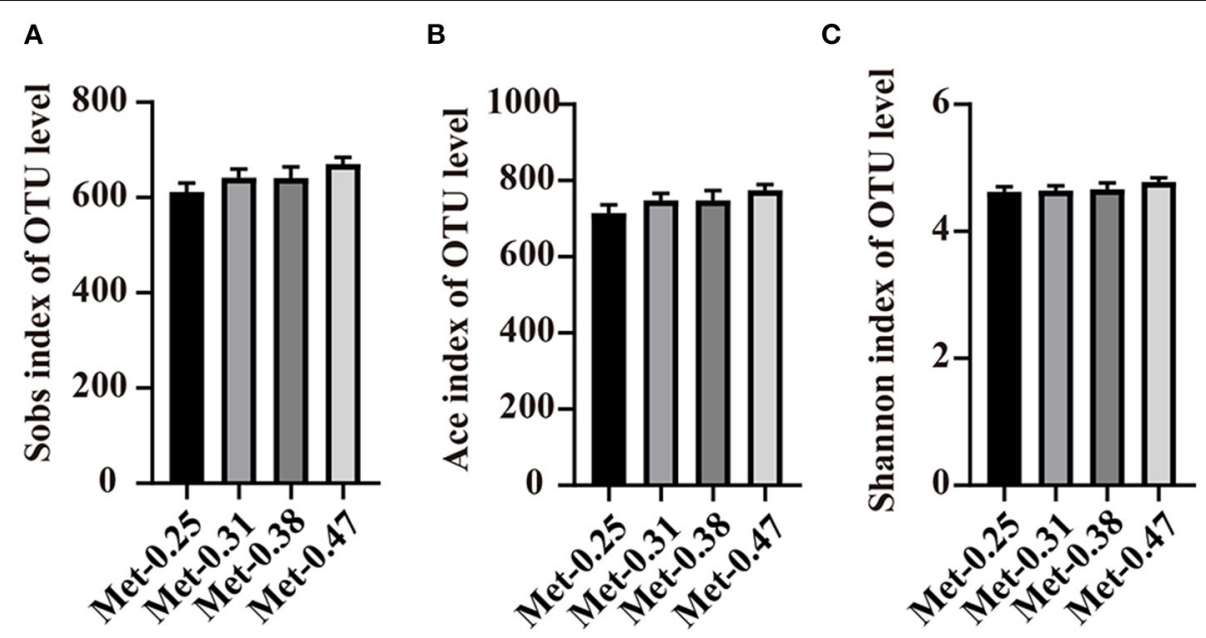

FIGURE 1 | The effects of different dietary methionine (Met) supplementation in low protein diets on the alpha diversity of the cecal microbiota in laying hens. (A) Sobs index of the community diversity. (B) Ace index of the community richness. (C) Shannon index of the community diversity. Data were presented as means \pm SEM $(n=$ 9 per group). Significant differences were tested by a Student's $t$-test.

\section{Effects of Different Levels of Met Supplementation on the Gut Microbial Composition and Function of Laying Hens With Low Protein Diets}

To investigate the effects of different Met levels in low protein diets on the gut microbial composition of laying hens, $16 \mathrm{~S}$ rDNA sequencing was performed. In the end, 2,154,182 sequences were obtained. Through a clustering operation, the sequences were divided into many groups according to $97 \%$ similarity. Each group was an OTU, and a total of 1,075 OTUs were obtained, which could be divided into 19 phyla, 34 classes, and 174 genera. The results of the alpha diversity analysis showed that there was no significant difference between the Sobs index, ACE index, and Shannon index among the four treatment groups (Figures 1A-C).

The relative abundance at the phylum and genus levels was studied. Notably, different levels of Met in diets changed the structure and composition of the intestinal microorganism of laying hens. From the perspective of the phylum level,
Bacteroidetes and Firmicutes are the two kinds of bacteria that account for the largest proportion in the cecum of laying hens, exceeding $95 \%$ of the total cecum bacteria. In addition, the proportion of Firmicute/Bacteroidetes in the $0.25 \%$ Met group was similar to that in the $0.47 \%$ Met group, but both were lower than that in the $0.38 \%$ Met group and higher than that in the $0.31 \%$ Met group (Figure 2A). At the genus level, Bacteroides, Rikenellaceae_RC9_gut_group, Lactobacillus, and Unclassified_O_Bacteroidales accounted for nearly $50 \%$ of the total cecal bacteria. Rikenellaceae_RC9_gut_group was the highest in the $0.31 \%$ Met group, followed by the 0.47 , 0.25 , and $0.38 \%$ Met groups. The relative abundance of Lactobacillus in the cecum of laying hens also increased with the increase of dietary Met level (Supplementary Table 3). The proportions of Bacteroides in all treatment groups was similar, with these proportions in the $0.25,0.31,0.38$, and $0.47 \%$ Met groups being 19.62, 17.37, 18.69, and 20.31\%, respectively (Figure 2B). The results of the PCoA showed that the gut microbiota in the $0.25,0.31,0.38$, and $0.47 \%$ Met groups were aggregated, respectively (Figure 2C). These results indicated 


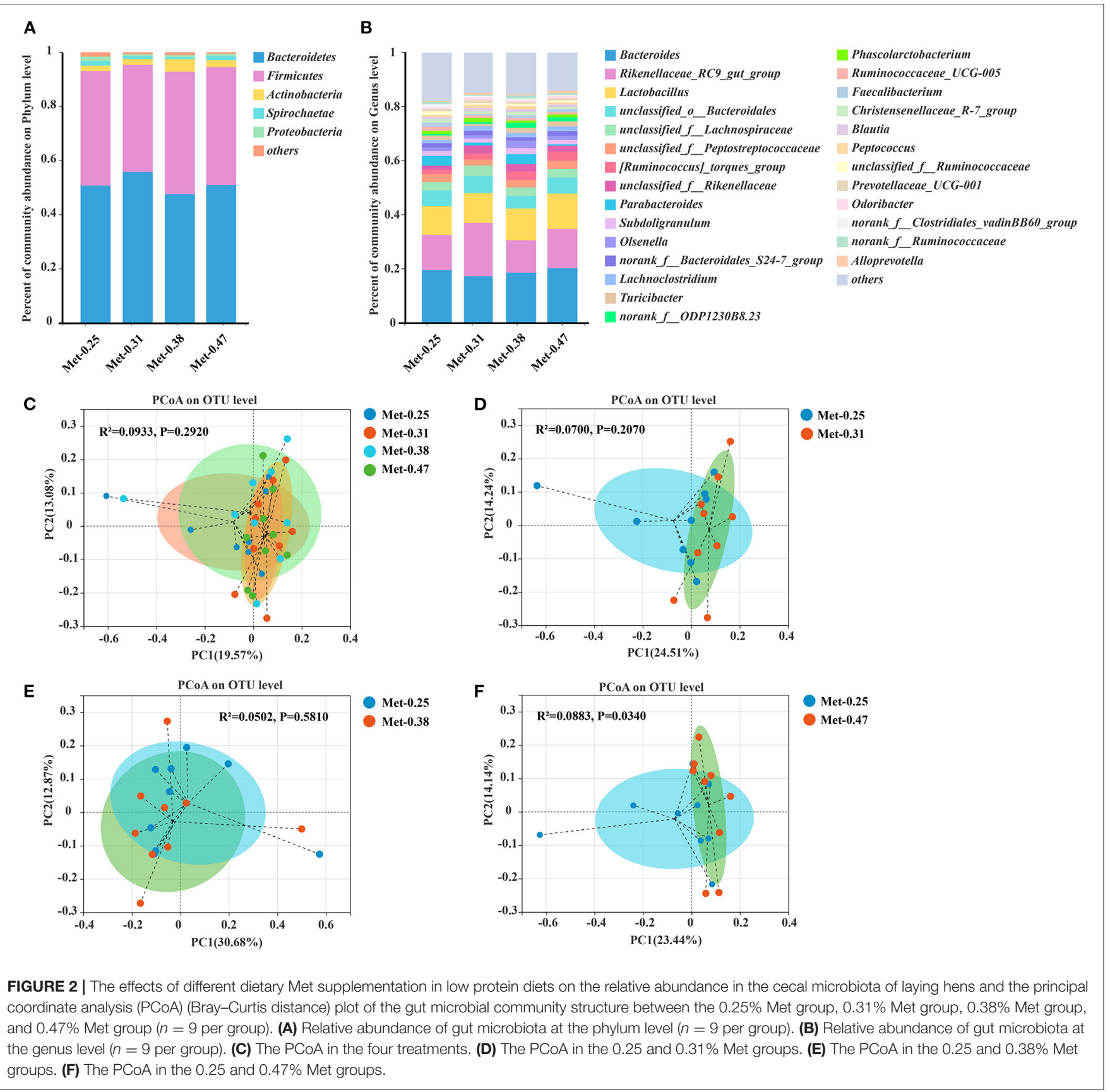

that different dietary Met levels changed the gut microbiota structure of laying hens to some extent. Moreover, compared with the distribution of spots in the $0.25 \%$ Met group, the distribution of spots in the 0.31 and $0.47 \%$ Met groups was more dispersed, and the distribution pattern of spots in the $0.38 \%$ Met group was similar to that in the $0.25 \%$ Met group (Figures 2D-F).

The LEfSe was used to figure out which bacterial taxa were statistically and biologically responsible for these differences. As shown in Figure 3, compared with the $0.25 \%$ Met group, [Eubacterium]_brachy_group,
unclassified_p_Bacteroidetes,

norank_c_OPB35_soil_group, Butyricimonas, Solobacterium, and Streptococcus were enriched in the $0.31 \%$ Met group (Figure 3A). Lachnoclostridium, [Eubacterium]_brachy_group, Faecalitalea, Faecalicoccus, Lachnospiraceae_UCG-002, Family_XIII_AD3011_group, and Enterococcus were enriched in the $0.38 \%$ Met group (Figure 3B). [Ruminococcus]_torques_group, Erysipelatoclostridium, [Eubacterium]_brachy_group, Brachyspira, Faecalicoccus, Faecalitalea, Coprococcus_1, [Clostridium]_innocuum_group, and Lachnospiraceae_FCSO20_group were enriched in the $0.47 \%$ Met group (Figure 3C). 


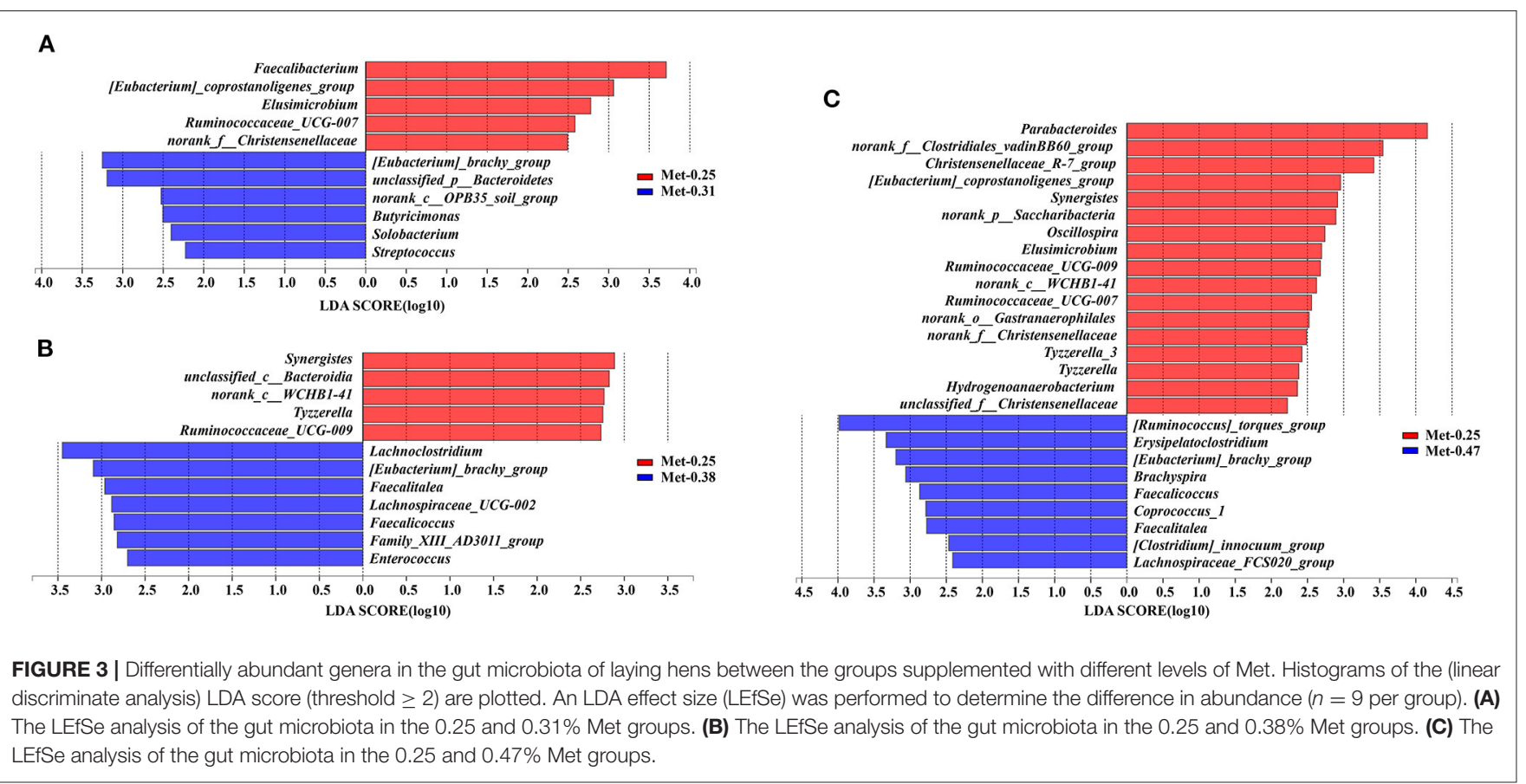

As shown in Supplementary Figures 1A,B, the different levels of dietary Met have significant effects on the intestinal microorganisms of laying hens. For example, compared with the other three groups, the $0.25 \%$ Met group has more significant effects on [Eubacterium]_coprostanoligenes_group, Ruminococcaceae_UCG-007, norank_f_Christensenellaceae, Elusimicrobium,

Ruminococcaceae_UCG-009, norank_c_WCHB1-41, and norank_o_Gastranaerophilales. In addition, Ruminococcaceae_UCG-009 and Norank_O_gastranaerophilales were affected significantly, and the $0.31 \%$ Met group has more significant effects on [Eubacterium]_brachy_group. The $0.38 \%$ Met group has more significant effects on norank_p_Saccharibacteria, Faecalitalea, and Family_XIII_AD3011_group, while the $0.47 \%$ Met group has more significant effects on Treponema_2, Coprococcus_1, Faecalicoccus, and [Clostridium]_innocuum_group (Supplementary Figures 1A,B). To elucidate the underlying molecular mechanism of Met in a low protein diet on the gut microbial function of hens, a KEGG pathway analysis were performed (Figure 4B). Interestingly, the most enriched pathways were closely related to carbohydrate metabolism, amino acid metabolism, energy metabolism, metabolism of cofactors and vitamins, and lipid metabolism.

\section{Correlation Between the Differential Microbes Induced by the Supplementation of Met in Low Protein Diets and Key Parameters}

In order to predict the correlation between the intestinal microbial communities and key parameters, an RDA and Spearman correlation matrix were performed. As shown in Figures 4A, 5, Ruminococcaceae_UCG-007, Ruminococcaceae_UCG-00, norank_o_Gastranaerophilales, and norank_c_WCHB1-41 were positively correlated with ADFI, EW, FBW, TG, EM, EP, and ADG, among which Ruminococcaceae_UCG-009 and Norank_o_gastranaerophilales were negatively correlated with FER $(p<0.05)$. On the contrary, Faecalitalea was negatively correlated with these traits $(p<0.05)$, while it was positively correlated with FER, but not significantly. Elusimicrobium was positively correlated with ADFI, EW, FBW, EM, EP, and ADG but negatively correlated with FER $(p<0.05)$. Norank_f_Christensenellaceae was significantly positively correlated with ADFI, EW, TG, $\mathrm{EM}$, and ADG, but negatively correlated with FER and UA $(p<0.05)$. Norank_P_Saccharibacteria was significantly positively correlated with EW, ADFI, FBW, and TG $(p<0.05)$. [Eubacterium]_coprostanoligenes_group was significantly positively correlated with EW $(p<0.05)$. In contrast, Faecalicoccus was significantly negatively correlated with ADFI, FBW, TG, EM, EP, and ADG $(p<0.01)$, but positively correlated with FER $(p<0.05)$. Family_xii_ad3011_group was negatively correlated with ADFI, but positively correlated with UA $(p<0.05)$. [Eubacterium]_Brachy_group was negatively correlated with ADFI, FBW, EM, EP, and ADG $(p<0.05)$. Coprococcus_1 was negatively correlated with EW and TG $(p<0.05)$. [Clostridium]_innocuum_group was significantly negatively correlated with FBW and TG $(p<0.05)$. There was a certain correlation between the production traits and other unmentioned intestinal microflora of laying hens, but their influence is not significant (Figure 5).

\section{DISCUSSION}

For a long time, Met has been used as a feed additive in livestock and poultry diets to maintain animal health and growth performance. According to previous studies, because of the 

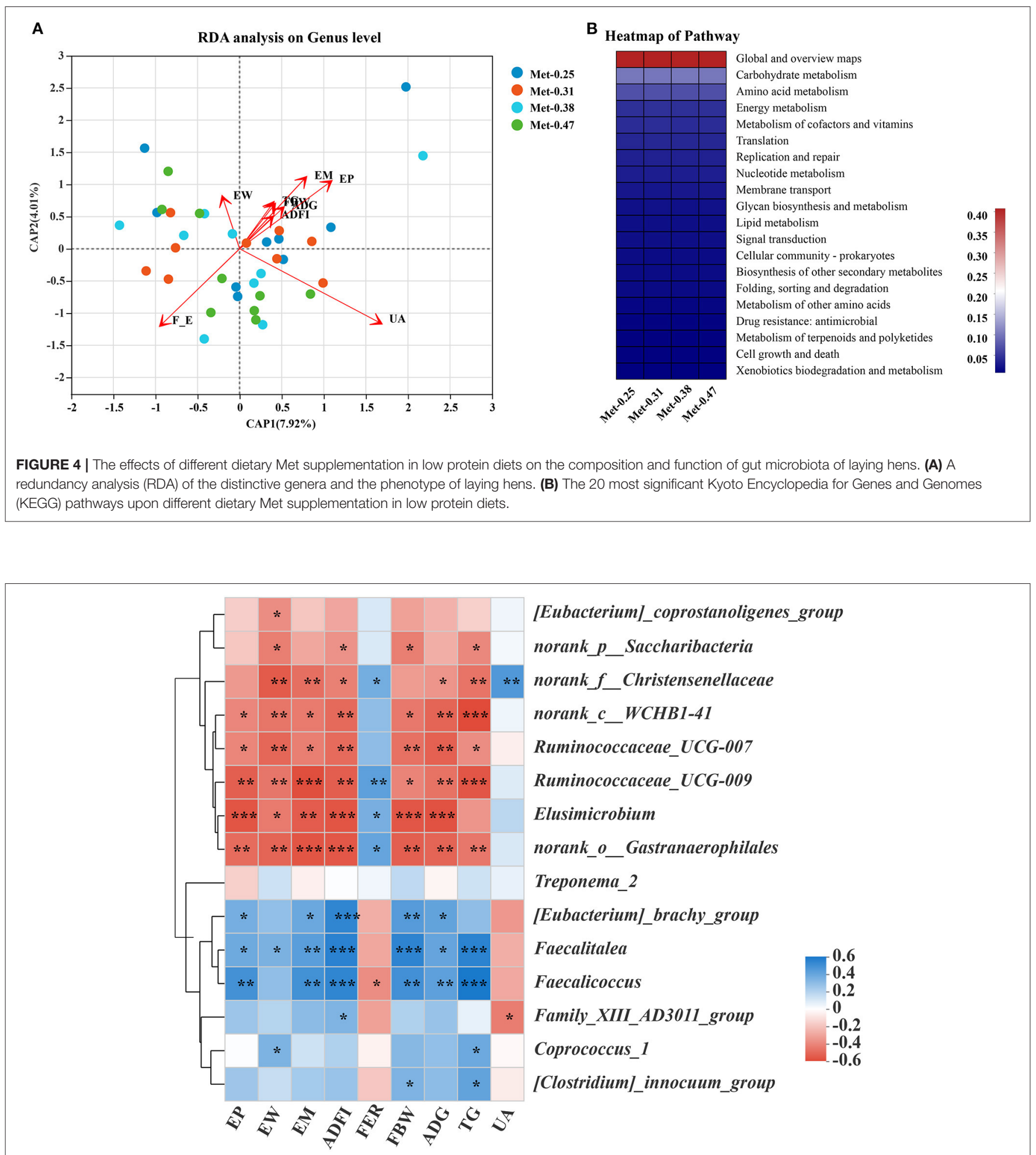

[Eubacterium]_coprostanoligenes_group

norank_p_saccharibacteria

norank_f_Christensenellaceae

norank_c_WCHB1-41

Ruminococcaceae_UCG-007

Ruminococcaceae_UCG-009

Elusimicrobium

norank_o_Gastranaerophilales

Treponema_2

[Eubacterium]_brachy_group

Faecalitalea

Faecalicoccus

Family_XIII_AD3011_group

Coprococcus_1

0.6

$-0.4$

$-0.2$

$-0$

$-0.2$

$-0.4$

[Clostridium]_innocuum_group

FIGURE 5 | The effects of different dietary Met supplementation in low protein diets on the difference of the gut microbiota and its correlation with the phenotype of laying hens. The correlation between the various gut microbiota and the phenotype of laying hens. Asterisks indicate significant correlations $\left({ }^{*} p \leq 0.05,{ }^{* *} p \leq 0.01\right.$, $\left.{ }^{\star \star *} p \leq 0.001\right)$. The blue represents a significantly positive correlation $(p \leq 0.05)$, the red represents a significantly negative correlation $(p \leq 0.05)$, and the white represents no significant correlation $(p>0.05)$. 
lack of essential amino acids in low protein diets, production performance and egg quality are negatively affected, the intestinal morphology is damaged, and the immune response level is reduced (41-43). In this study, Peking Pink laying hens were selected as the experimental model to explore the effects of different levels of Met in the low protein diet on the performance, egg quality, serum biochemical indexes, and gut microbial composition of the laying hens. Our results showed that the supplementation of Met in the low protein diet could significantly improve various indices of laying hens.

When the dietary protein level is reduced to $13-14 \%$, the performance of laying hens will be directly affected if the synthetic amino acids are not supplemented in time $(44,45)$. Keshavarz showed that the addition of synthetic amino acids to a low protein diet could effectively improve the performance of laying hens and reduce nitrogen emissions, which is also consistent with the results of others (46-48). From these, we can understand the significance of additional amino acids in the low protein diet, which is the same as the results in this experiment.

Studies have found that, if the Met intake of laying hens was increased, then EW would increase. Meanwhile, laying performance would be significantly improved, but with a decreased FER $(19,49)$. Similarly, we obtained the quadratic equation between Met and sulfur-containing amino acids and EP, EW, and FER. Within a certain range, the increase of Met level can increase EP and EM, while decreasing FER. These results indicated that the supplementation of Met in diets promotes the feeding and growth of laying hens and improves feed conversion ratio, which were also similar with Esteve (50). In our experiment, with the increase of Met, egg weight also increased at each stage. However, different Met levels had no significant effects on eggshell color, strength, Haugh unit, and other indices.

A number of indicators in the serum often reflect the health status of animals. When animals are in a healthy state, protein synthesis increases along with TP and ALB. In our experiment, TP and ALB in the 0.38 and $0.47 \%$ Met groups were higher than those in the other Met groups. The IgA in the $0.47 \%$ Met group was higher than that in the other three groups, which was similar to the research results of Liu et al. (51). Those indicated that the metabolism of laying hens in the $0.47 \%$ Met group was more vigorous, and the level of immune response was higher. As one of the end-products of protein metabolism in poultry, UA is usually used as an indicator to measure the requirement of amino acids. It reflects the level of protein metabolism in animals (52). Our results showed that the changes of UA were stable when the Met level was in the range of $0.31-0.47 \%$, and UA was lower than that in the $0.25 \%$ Met group. Therefore, it could be inferred that the amino acids in laying hens in the three groups with higher Met levels were more fully utilized.

Dietary energy levels are the main nutritional factors affecting egg quality (53). Our results showed that there was a positive linear correlation between the dietary Met level and TG, which was similar to the difference in EW among all groups. We postulated that a higher TG in serum could synthesize more fat and fully meet the energy needs of laying hens, thus affecting EW.
A certain amount of abdominal fat storage is beneficial to prevent insufficient energy intake from affecting laying performance (54), and, based on the results of the $0.25 \%$ Met group, we inferred that when the Met in the diet is insufficient, the liver metabolism of laying hens is disturbed and the fat transport is obstructed, leading to fatty liver disease in the end.

Normal and stable microflora is an important prerequisite to ensure the health of poultry. Intestinal microflora is involved in the metabolism and growth of the host, and also affects feed conversion and nutrient digestion and absorption by changing the intestinal tissue morphology of poultry, thus affecting their performance (55-57). Our results showed that, at the genus level, Bacteroides, Rikenellaceae_RC9_gut_group, Lactobacillus, and unclassified_o_Bacteroidales were the dominant genera accounting for nearly $50 \%$ of the total cecal bacteria of laying hens. In the meantime, the Lactobacillus proportion increased with the increase of Met. Yan used a metagenomic analysis technology to find that Lactobacillus could promote the absorption of nutrients and improve the feeding efficiency of poultry (58). Some scholars have found that Lactobacillus and Bifidobacterium can synthesize a variety of VB beneficial to animals by participating in their metabolism. It can also convert mineral elements into ions that are easily absorbed by animals to improve their utilization rate (59). In contrast to the change in the proportion of Lactobacillus, the proportion of Faecalibacterium decreases with the increase of Met in the diet. Faecalitalea and Faecalicoccus of Clostridium had significantly negative effects on ADFI, FBW, TG, EM, EP, ADG, and other indices, but had positive effects on FER. Other studies have shown that Clostridia has an adverse effect on animal performance (60), and our results are consistent with this finding. We can conclude that the supplementation of Met in diets is beneficial to laying hens. In conclusion, it can be inferred that Met in low protein diets may improve the intestinal morphology and production performance of laying hens by promoting beneficial bacteria proliferation and competitively inhibiting harmful bacteria proliferation or infection.

\section{CONCLUSION}

The use of the low protein diet could alleviate the current situation of the raw material shortage of protein feed and reduce the nitrogen emission from the feces and urine of livestock and poultry to reduce environmental pollution. Adding Met to the low protein diet could have positive effects on the production performance, reproductive system, host metabolism, and gut microbial composition of laying hens. For example, the addition of Met increased the abundance of Lactobacillus and decreased the proportion of Faecalibacterium in the gut. Meanwhile, there were also significant correlations between the gut microbiota and traits of laying hens. Specifically, the proportion of Faecalicoccus was significantly positively correlated with FER, but negatively correlated with ADFI, FBW, ADG, and other traits. At present, there are few studies on the effects of low protein diets on the gut microflora of laying hens. We hoped that our study will fill in some gaps in this field. 


\section{DATA AVAILABILITY STATEMENT}

The datasets presented in this study can be found in online repositories. The names of the repository/repositories and accession number(s) can be found at: https://www.ncbi.nlm.nih. gov/, PRJNA745253.

\section{ETHICS STATEMENT}

The animal study was reviewed and approved by China Agricultural University Animal Care and Use Committee (AW32301202-2-1, Beijing, China).

\section{AUTHOR CONTRIBUTIONS}

MM, QM, JZ, and SG designed the study. MM, SG, and SH conducted the experiments, drafted the manuscript, polished the manuscript, and finished the submission. MM, ML, and SG guided the analysis of the experimental data. QM, LZ, and JZ helped with revisiting and reviewing the manuscript. All authors read and approved the final manuscript.

\section{FUNDING}

This study was supported by the National Science Foundation of China (Grant No. 31772621), a Special Fund for China

\section{REFERENCES}

1. Ravindran V, Blair R. Feed resources for poultry production in Asia and the Pacific. III Animal protein sources. World's Poult Sci J. (2007) 49:21935. doi: 10.1079/WPS19930020

2. Cao Y, Li D. Impact of increased demand for animal protein products in Asian countries: implications on global food security. Anim Front. (2013) 3:48-55. doi: 10.2527/af.2013-0024

3. Tomé D, Bos C. Dietary protein and nitrogen utilization. J Nutr. (2000) 130:1868s-73s. doi: 10.1093/jn/130.7.1868S

4. Tome D. Criteria and markers for protein quality assessment - a review. $\mathrm{Br} J$ Nutr. (2012) 108(Suppl.2):S222-9. doi: 10.1017/S0007114512002565

5. Kerr BJ, Ziemer CJ, Trabue SL, Crouse JD, Parkin TB. Manure composition of swine as affected by dietary protein and cellulose concentrations. J Anim Sci. (2006) 84:1584-92. doi: 10.2527/2006.8461584x

6. Aarnink AJA, Verstegen MWA. Nutrition, key factor to reduce environmental load from pig production. Livest Sci. (2007) 109:194-203. doi: 10.1016/j.livsci.2007.01.112

7. Hristov AN, Hanigan M, Cole A, Todd R, Mcallister TA, Ndegwa PM, et al. Review: ammonia emissions from dairy farms and beef feedlots. Can J Anim Ence. (2011) 91:1-35. doi: 10.4141/CJAS10034

8. Wang Y, Zhou J, Wang G, Cai S, Zeng X, Qiao S. Advances in low-protein diets for swine. J Anim Sci Biotechnol. (2018) 9:60. doi: 10.1186/s40104-018-0276-7

9. Nahm KH. Feed formulations to reduce $\mathrm{N}$ excretion and ammonia emission from poultry manure. Bioresour Technol. (2007) 98:2282300. doi: 10.1016/j.biortech.2006.07.039

10. Kamran Z, Sarwar M, Nisa M, Nadeem MA, Mahmood S, Babar ME, et al. Effect of low-protein diets having constant energy-to-protein ratio on performance and carcass characteristics of broiler chickens from one to thirty-five days of age. Poult Sci. (2008) 87:468-74. doi: 10.3382/ps.2007-00180

11. Hernandez F, Megias M, Orengo J, Martinez S, Madrid J. Effect of dietary protein level on retention of nutrients, growth performance, litter
Agricultural Research System program (CARS-40-K08), National Key Research and Development Program of China (2017YFD0500500), and the Special Fund from Chinese Universities Scientific Fund (2018TC043).

\section{ACKNOWLEDGMENTS}

We thank all the technicians in the experimental animal facility of the China Agricultural University for providing the daily care of the laying hens. We also thank Shanghai Majorbio Bio-Pharm Technology Co. for their bioinformatic analysis of the data.

\section{SUPPLEMENTARY MATERIAL}

The Supplementary Material for this article can be found online at: https://www.frontiersin.org/articles/10.3389/fnut.2021. 739676/full\#supplementary-material

Supplementary Figure 1 | The effects of different dietary Met supplementation in low protein diets on the difference of the gut microbiota of laying hens. (A) The LEfSe analysis of the gut microbiota in the four treatments. (B) The mean proportion of the gut microbiota in the four treatments.

Supplementary Table 1 | Ingredients and nutrient content of the diets (\% DM).

Supplementary Table 2 | Regression equation between production performance and Met and sulfur-containing amino acid intake.

Supplementary Table 3 | The proportion of gut microbiota within the four Met groups on genus level.

composition and $\mathrm{NH} 3$ emission using a multi-phase feeding programme in broilers. Span J Agri Res. (2013) 11:736-46. doi: 10.5424/sjar/2013113-3597

12. Zhang S, Zeng X, Ren M, Mao X, Qiao S. Novel metabolic and physiological functions of branched chain amino acids: a review. J Anim Sci Biotechnol. (2017) 8:10. doi: 10.1186/s40104-016-0139-Z

13. Nie C, He T, Zhang W, Zhang G, Ma X. Branched chain amino acids: beyond nutrition metabolism. Int J Mol Sci. (2018) 19:40954. doi: 10.3390/ijms19040954

14. Graciene CS, Edivaldo AG, Javer AVF. Performance of Japanese quails fed diets with low-protein and isoleucine. Acta Sci Anim Sci. (2016) 38:29533. doi: 10.4025 /actascianimsci.v38i2.29533

15. Deng D, Huang RL, Li TJ, Wu GY, Xie MY, Tang ZR, et al. Nitrogen balance in barrows fed low-protein diets supplemented with essential amino acids. Livestock Sci. (2007) 109:220-3. doi: 10.1016/j.livsci.2007.01.122

16. Wu L, He LQ, Cui ZJ, Liu G, Yao K, Wu F, et al. Effects of reducing dietary protein on the expression of nutrition sensing genes (amino acid transporters) in weaned piglets. J Zhejiang Univ Sci B. (2015) 16:496502. doi: 10.1631/jzus.B1400259

17. Ospina-Rojas IC, Murakami AE, Duarte CR, Eyng C, Oliveira CA, Janeiro V. Valine, isoleucine, arginine and glycine supplementation of low-protein diets for broiler chickens during the starter and grower phases. Br Poult Sci. (2014) 55:766-73. doi: 10.1080/00071668.2014.970125

18. Awad EA, Zulkifli I, Soleimani AF, Aljuobori A. Effects of feeding male and female broiler chickens on low-protein diets fortified with different dietary glycine levels under the hot and humid tropical climate. Ital J Anim Sci. (2017) 16:453-61. doi: 10.1080/1828051X.2017.1291288

19. Ratriyanto A, Indreswari R, Nuhriawangsa A, Purwanti E. Feed efficiency of diets with different energy and protein concentrations supplemented with methionine in laying quails. Iop Conference. (2018) 142. doi: 10.1088/1755-1315/142/1/012001

20. Bauchart-Thevret C, Stoll B, Burrin DG. Intestinal metabolism of sulfur amino acids. Nutr Res Rev. (2009) 22:175-87. doi: 10.1017/S0954422409990138 
21. Chen Y, Li D, Dai Z, Piao X, Wu Z, Wang B, et al. L-methionine supplementation maintains the integrity and barrier function of the smallintestinal mucosa in post-weaning piglets. Amino Acids. (2014) 46:113142. doi: 10.1007/s00726-014-1675-5

22. Chen YP, Cheng YF, Li XH, Yang WL, Wen C, Zhuang S, et al. Effects of threonine supplementation on the growth performance, immunity, oxidative status, intestinal integrity, and barrier function of broilers at the early age. Poult Sci. (2017) 96:405-13. doi: 10.3382/ps/pew240

23. Zeitz JO, Kaltenböck S, Most E, Eder K. Effects of L-methionine on performance, gut morphology and antioxidant status in gut and liver of piglets in relation to DL-methionine. J Anim Physiol Anim Nutr. (2019) 103:242-50. doi: 10.1111/jpn.13000

24. Jariyahatthakij P, Chomtee B, Poeikhampha T, Loongyai W, Bunchasak C. Effects of adding methionine in low-protein diet and subsequently fed lowenergy diet on productive performance, blood chemical profile, and lipid metabolism-related gene expression of broiler chickens. Poult Sci. (2018) 97:2021-33. doi: 10.3382/ps/pey034

25. Bunchasak C. Role of dietary methionine in poultry production. J Poult Sci. (2009) 46:169-79. doi: 10.2141/jpsa.46.169

26. Rama Rao SV, Praharaj NK, Ramasubba Reddy V, Panda AK. Interaction between genotype and dietary concentrations of methionine for immune function in commercial broilers. Br Poult Sci. (2003) 44:104-12. doi: 10.1080/0007166031000085283

27. Maroufyan E. The effect of methionine and threonine supplementations on immune responses of broiler chickens challenged with infectious bursal disease. Am J Appl Sci. (2010) 7:50. doi: 10.3844/ajassp.2010.44.50

28. Xie M, Hou SS, Huang W, Fan HP. Effect of excess methionine and methionine hydroxy analogue on growth performance and plasma homocysteine of growing Pekin ducks. Poult Sci. (2007) 86:1995-9. doi: 10.1093/ps/86.9.1995

29. Zhang YN, Xu RS, Min L, Ruan D, Kim HY, Hong YG, et al. Effects of L-methionine on growth performance, carcass quality, feather traits, and small intestinal morphology of Pekin ducks compared with conventional DL-methionine. Poult Sci. (2019) 98:6866-72. doi: 10.3382/ps/pez438

30. Schutte JB, De Jong J, Smink W, Pack M. Replacement value of betaine for DL-methionine in male broiler chicks. Poult Sci. (1997) 76:3215. doi: $10.1093 / \mathrm{ps} / 76.2 .321$

31. Ding W, Smulan LJ, Hou NS, Taubert S, Watts JL, Walker AK. s-Adenosylmethionine levels govern innate immunity through distinct methylation-dependent pathways. Cell Metab. (2015) 22:633-45. doi: 10.1016/j.cmet.2015.07.013

32. Edgar RC. UPARSE: highly accurate OTU sequences from microbial amplicon reads. Nat Methods. (2013) 10:996-8. doi: 10.1038/nmeth.2604

33. Li N, Huang S, Jiang L, Dai Z, Li T, Han D, et al. Characterization of the early life microbiota development and predominant lactobacillus species at distinct gut segments of low- and normal-birth-weight piglets. Front Microbiol. (2019) 10:797. doi: 10.3389/fmicb.2019.00797

34. Edgar RC, Haas BJ, Clemente JC, Quince C, Knight R. UCHIME improves sensitivity and speed of chimera detection. Bioinformatics. (2011) 27:2194200. doi: 10.1093/bioinformatics/btr381

35. Schloss PD, Westcott SL, Ryabin T, Hall JR, Hartmann M, Hollister EB, et al. Introducing mothur: open-source, platform-independent, communitysupported software for describing and comparing microbial communities. Appl Environ Microbiol. (2009) 75:7537-41. doi: 10.1128/AEM.01541-09

36. Huang SM, Wu ZH, Li TT, Liu C, Han DD, Tao SY, et al. Perturbation of the lipid metabolism and intestinal inflammation in growing pigs with low birth weight is associated with the alterations of gut microbiota. Sci Total Environ. (2020) 719:137382. doi: 10.1016/j.scitotenv.2020.137382

37. Koistinen VM, Kärkkäinen O, Borewicz K, Zarei I, Jokkala J, Micard V, et al. Contribution of gut microbiota to metabolism of dietary glycine betaine in mice and in vitro colonic fermentation. Microbiome. (2019) 7:103. doi: 10.1186/s40168-019-0718-2

38. Zhai Q, Cen S, Jiang J, Zhao J, Zhang H, Chen W. Disturbance of trace element and gut microbiota profiles as indicators of autism spectrum disorder: a pilot study of Chinese children. Environ Res. (2019) 171:5019. doi: 10.1016/j.envres.2019.01.060

39. Phavichitr N, Wang S, Chomto S, Tantibhaedhyangkul R, Kakourou $A$, Intarakhao $S$, et al. Impact of synbiotics on gut microbiota during early life: a randomized, double-blind study. Sci Rep. (2021) 11:3534. doi: 10.1038/s41598-021-83009-2

40. Langille MG, Zaneveld J, Caporaso JG, Mcdonald D, Knights D, Reyes JA, et al. Predictive functional profiling of microbial communities using 16S rRNA marker gene sequences. Nat Biotechnol. (2013) 31:81421. doi: $10.1038 /$ nbt. 2676

41. Torki M, Mohebbifar A, Ghasemi HA, Zardast A. Response of laying hens to feeding low-protein amino acid-supplemented diets under high ambient temperature: performance, egg quality, leukocyte profile, blood lipids, and excreta pH. Int J Biometeorol. (2015) 59:575-84. doi: 10.1007/s00484-014-0870-0

42. Geng S, Huang S, Ma Q, Li F, Gao Y, Zhao L, et al. Alterations and correlations of the gut microbiome, performance, egg quality, and serum biochemical indexes in laying hens with low-protein amino acid-deficient diets. ACS Omega. (2021) 6:13094-104. doi: 10.1021/acsomega.1c00739

43. Kidd MT, Maynard CW, Mullenix GJ. Progress of amino acid nutrition for diet protein reduction in poultry. J Anim Sci Biotechnol. (2021) 12:45. doi: 10.1186/s40104-021-00568-0

44. Gunawardana P, Roland DA, Bryant MM. Effect of energy and protein on performance, egg components, egg solids, egg quality, and profits in molted Hy-line W-36 hens. J Appl Poult Res. (2008) 17:432-9. doi: 10.3382/japr.2007-00085

45. Khajali F, Khoshouie EA, Dehkordi SK, Hematian M. Production performance and egg quality of Hy-line W36 laying hens fed reduced-protein diets at a constant total sulfur amino acid:lysine ratio. J Appl Poult Res. (2008) 17:390-7. doi: 10.3382/japr.2008-00002

46. Keshavarz K, Austic RE. The use of low-protein, low-phosphorus, amino acidand phytase-supplemented diets on laying hen performance and nitrogen and phosphorus excretion. Poult Sci. (2004) 83:75-83. doi: 10.1093/ps/83.1.75

47. Saima KM, Jabbar MA, Mehmud A, Mahmood MMA. Effect of lysine supplementation in low protein diets on the performance of growing broilers. Pak Vet J. (2010) 30.

48. Arboleya S, Binetti A, Salazar N, Fernández N, Solís G, HernándezBarranco A, et al. Establishment and development of intestinal microbiota in preterm neonates. FEMS Microbiol Ecol. (2012) 79:763-72. doi: 10.1111/j.1574-6941.2011.01261.x

49. Harms RH, Miles RD. Influence of fermacto on the performance of laying hens when fed diets with different levels of methionine. Poult Sci. (1988) 67:842-4. doi: 10.3382/ps.0670842

50. Esteve-Garcia E, Khan DR. Relative bioavailability of DL and L-methionine in broilers. Open J Anim Sci. (2018) 8:151-62. doi: 10.4236/ojas.2018.82011

51. Liu Y, Lin X, Zhou X, Wan D, Wang Z, Wu X, et al. Effects of dynamic feeding low and high methionine diets on egg quality traits in laying hens. Poult Sci. (2017) 96:1459-65. doi: 10.3382/ps/pew398

52. Featherston WR. Nitrogenous metabolites in the plasma of chicks adapted to high protein diets. Poult Sci. (1969) 48:646-52. doi: 10.3382/ps.0480646

53. Kang HK, Park SB, Jeon JJ, Kim HS, Park KT, Kim SH, et al. Effect of increasing levels of apparent metabolizable energy on laying hens in barn system. Asian-Australas J Anim Sci. (2018) 31:1766-72. doi: 10.5713/ajas.17. 0846

54. Murugesan GR, Persia ME. Validation of the effects of small differences in dietary metabolizable energy and feed restriction in first-cycle laying hens. Poult Sci. (2013) 92:1238-43. doi: 10.3382/ps.2012-02719

55. Pan D, Yu Z. Intestinal microbiome of poultry and its interaction with host and diet. Gut Microbes. (2014) 5:108-19. doi: 10.4161/gmic.26945

56. Chang EB, Martinez-Guryn K. Small intestinal microbiota: the neglected stepchild needed for fat digestion and absorption. Gut Microbes. (2019) 10:235-40. doi: 10.1080/19490976.2018.1502539

57. Rastelli M, Cani PD, Knauf C. The gut microbiome influences host endocrine functions. Endocr Rev. (2019) 40:1271-84. doi: 10.1210/er.2018-00280

58. Yan W, Sun C, Yuan J, Yang N. Gut metagenomic analysis reveals prominent roles of Lactobacillus and cecal microbiota in chicken feed efficiency. Sci Rep. (2017) 7:45308. doi: 10.1038/srep45308

59. Leblanc JG, Milani C, De Giori GS, Sesma F, Van Sinderen D, Ventura M. Bacteria as vitamin suppliers to their host: a gut microbiota perspective. Curr Opin Biotechnol. (2013) 24:160-8. doi: 10.1016/j.copbio.2012.08.005

60. Shindo Y, Dobashi Y, Sakai T, Monma C, Miyatani H, Yoshida Y. Epidemiological and pathobiological profiles of Clostridium perfringens 
infections: review of consecutive series of 33 cases over a 13-year period. Int $J$ Clin Exp Pathol. (2015) 8:569-77.

Conflict of Interest: The authors declare that the research was conducted in the absence of any commercial or financial relationships that could be construed as a potential conflict of interest.

Publisher's Note: All claims expressed in this article are solely those of the authors and do not necessarily represent those of their affiliated organizations, or those of the publisher, the editors and the reviewers. Any product that may be evaluated in this article, or claim that may be made by its manufacturer, is not guaranteed or endorsed by the publisher.

Copyright $\odot 2021$ Ma, Geng, Liu, Zhao, Zhang, Huang and Ma. This is an openaccess article distributed under the terms of the Creative Commons Attribution License (CC BY). The use, distribution or reproduction in other forums is permitted, provided the original author(s) and the copyright owner(s) are credited and that the original publication in this journal is cited, in accordance with accepted academic practice. No use, distribution or reproduction is permitted which does not comply with these terms. 